\title{
Negative DEP traps for single cell immobilisation $\uparrow$
}

\author{
Rupert S. Thomas, * Hywel Morgan* and Nicolas G. Green* \\ Received 4th November 2008, Accepted 10th February 2009 \\ First published as an Advance Article on the web 6th March 2009 \\ DOI: $10.1039 / \mathrm{b819267g}$
}

We present a novel design of micron-sized particle trap that uses negative dielectrophoresis (nDEP) to trap cells in high conductivity physiological media. The design is scalable and suitable for trapping large numbers of single cells. Each trap has one electrical connection and the design can be extended to produce a large array. The trap consists of a metal ring electrode and a surrounding ground plane, which create a closed electric field cage in the centre. The operation of the device was demonstrated by trapping single latex spheres and HeLa cells against a moving fluid. The dielectrophoretic holding force was determined experimentally by measuring the displacement of a trapped particle in a moving fluid. This was then compared with theory by numerically solving the electric field for the electrodes and calculating the trapping force, demonstrating good agreement. Analysis of the $80 \mu \mathrm{m}$ diameter trap showed that a $15.6 \mu \mathrm{m}$ diameter latex particle could be held with a force of $23 \mathrm{pN}$ at an applied voltage of $5 \mathrm{~V}$ peak-peak.

\section{Introduction}

Lab-on-a-chip (LOC) devices have emerged as a useful platform for cell studies. ${ }^{1}$ Cells can be manipulated in LOC systems using optical methods, ${ }^{2}$ hydrodynamics ${ }^{3}$ and electric fields, in particular dielectrophoresis (DEP). ${ }^{4,5}$ DEP is the movement of cells in non-uniform electric fields, and is now widely used for general cell manipulation, sorting and analysis in micro-fluidic devices. ${ }^{6-8}$ DEP devices have applications for cell separation (selective trapping of cells), observation of cellular response to stimuli (addition of drug) or cell culture on chip. In order to ensure longterm cell viability and minimise stress, it is desirable to keep cells suspended in their native culture medium, as this contains the required nutrients and is also osmotically balanced. The high electrical conductivity of such media restricts the use of some dielectrophoretic manipulation techniques, since positive DEP usually does not occur. In addition, positive DEP would result in the cells being attracted to high field points (e.g. electrode edges $)^{4,9,10}$ where cells can be lysed. ${ }^{11}$ Negative DEP occurs when the cell is less polarisable than the suspending medium, and describes the movement of particles away from high field regions. This is preferable to positive DEP for cell handling, because the cells are trapped away from high field regions and are less likely to experience large transmembrane potentials that could develop in the high-field regions near the electrodes.

One promising advantage of LOC is the ability to manipulate individual cells. ${ }^{12,13}$ Analysis of single cells allows the characteristics of rare or unusual cells to be measured, without the averaging effect that is present when whole populations are analysed.

The Nano Group, University of Southampton, Southampton, UK. E-mail: hm@ecs.soton.ac.uk

$\uparrow$ Electronic supplementary information (ESI) available: Additional information on the construction of the device, the fluid flow analysis used in the paper and a comprehensive set of numerical simulations on the design of the ring array and what assumptions are justified in those numerical simulations; and videos (ring trap beads and ring trap HeLa cells). See DOI: 10.1039/b819267g
A fast and reliable method of single cell manipulation should also enable the concentration of rare cells from a mixed population, which would be useful for purification of cell samples ex vivo. One route to single cell manipulation and analysis is the isolation of cells by confinement inside a particle trap. Negative DEP has been used to hold cells and particles at fixed positions inside potential energy wells, trapping them in free space at electric field minima, ${ }^{14,15}$ a result demonstrated in the four and eight electrode cages developed by Schnelle et al. ${ }^{16}$

An ideal dielectrophoretic cell trap should have the following characteristics:

- Operate in (high conductivity) physiological media.

- Have minimum power dissipation (avoid fluid heating).

- Limit the exposure of cells to high electric fields.

- Operate at high frequencies to minimise induced transmembrane potentials.

- Capture a single cell in a closed cage.

- Be scalable to an array, ideally with a single wire connection per trap.

The quadrupole and octopole trap have many of the above features: ${ }^{16}$ cells are levitated above a surface in $\mathrm{nDEP}$ cages and the cells are not subject to excessive trans-membrane potentials. Unfortunately, the number of electrical connections required per trap (4 or 8 respectively) and the required spatial configuration means that it is difficult to connect large numbers of independently controllable traps without many layers of interconnects. In order to overcome some of these issues, variants on the original quadrupole trap have been developed as large arrays, fabricated on silicon using CMOS technology. ${ }^{15}$ Combinations of different electrodes can be switched on and off to dynamically create traps, or to move particles around on the surface. In addition, automated control of these arrays has been demonstrated using integrated optical sensors - important as the number of traps is increased. ${ }^{15,17}$ Negative DEP traps for patterning single particles have also been demonstrated by the Voldman group. ${ }^{14,18}$ They fabricated planar microelectrodes to create potential energy 'microwells' for trapping single cells in 
physiological media against a surface. Such traps were not designed to be individually addressable, as multiple traps are connected in series, but this would be possible with some development of the electrical interconnections.

We have developed a novel and simple nDEP trap which meets all of the above design requirements, and is shown schematically in Fig. 1A. The trap or cage consists of a single metal ring electrode, driven by an AC signal, and separated by a uniform gap from a surrounding ground plane. The high field is found at the gap between the two electrodes and there is a field minimum in the centre of the ring electrode, resulting in a nDEP trap. Furthermore, the circular design ensures that this field minimum is three dimensional and the trap is closed in the vertical direction, meaning that this is in fact a field cage. Particles trapped in this region are held down on the surface and other particles are prevented from entering from any direction. Such a design can be fabricated using photolithography, but it requires an inter-layer dielectric insulator between the two metal layers, as shown by the exploded view of the three layers in Fig. 1A. A single cage has only one wire, and the device architecture is scalable (Fig. 1B), with the potential to create a large array of cages, with each cage individually switchable.
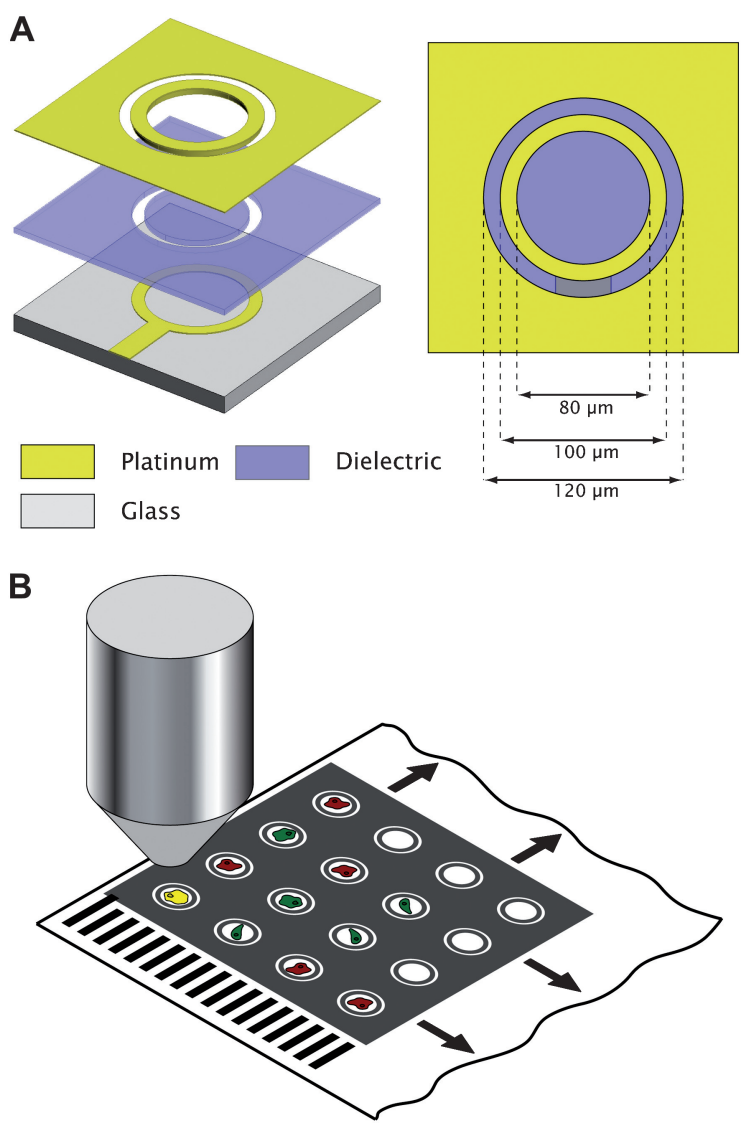

Fig. 1 (A) The ring traps were fabricated from two titanium/platinum layers with a benzocyclobutene (BCB) dielectric. An alternative design was also produced with dimensions 40/60/80 $\mu \mathrm{m}$. (B) Ideally, cell traps can be scaled into larger arrays. A single cell is trapped in each ring, and observed optically through a microscope. Cells can be maintained on chip for further culture, or released and removed from the chip by fluid flow.
This paper presents detail of the design and simulations of the dielectrophoretic characteristics of the cage and illustrates the principles of operation. Experimental measurements are made of the performance of the device and the response of particles inside the cage. The operation of the field cages as an array technology is then demonstrated on a range of different particles.

\section{Theory}

There are two physical effects on the particles held in the electric field cages: the dielectrophoretic force and the viscous drag from the suspending medium.

\section{Dielectrophoresis}

The theory of dielectrophoresis is well known and can be found in a number of books and reviews. ${ }^{19-22} \mathrm{~A}$ spatially non-uniform electric field gives rise to force on a polarisable particle. The timeaveraged force in the dipole approximation is:

$$
\left\langle\mathbf{F}_{D E P}\right\rangle=\pi a^{3} \varepsilon_{m} \operatorname{Re}\left(f_{C M}\right) \nabla|\mathbf{E}|^{2}
$$

where $a$ is the particle radius, $\varepsilon_{m}$ is the permittivity of the suspending medium, $\operatorname{Re}\left(f_{C M}\right)$ is the real part of the Clausius-Mossotti $(C M)$ factor for the particle and the surrounding media and $\mathbf{E}$ is the electric field. The Clausius-Mossotti factor describes the frequency dependence of the effective polarisability and for a spherical, homogeneous particle is:

$$
f_{C M}=\frac{\varepsilon_{p}^{*}-\varepsilon_{m}^{*}}{\varepsilon_{p}^{*}+2 \varepsilon_{m}^{*}}
$$

with a general complex permittivity

$$
\varepsilon^{*}=\varepsilon-j \frac{\sigma}{\omega}
$$

The subscripts $p$ and $m$ refer to particle or medium respectively, $\sigma$ is the conductivity and $\omega$ the angular frequency of the applied electric field. If the particle is more polarisable than the surrounding media, then $\operatorname{Re}\left(f_{C M}\right)$ is positive and the DEP force directs the particle towards regions of high electric field strength (generally towards the electrodes), positive dielectrophoresis (pDEP). Conversely, if the medium is more polarisable than the particle, $\operatorname{Re}\left(f_{C M}\right)$ is negative and the DEP force directs the particle towards regions of low electric field strength (generally away from the electrodes), negative dielectrophoresis (nDEP).

\section{Hydrodynamic effects}

A particle suspended in a moving liquid experiences a drag force proportional to the difference in their velocity vectors. The hydrodynamic drag force on a spherical body with low Reynolds number in a uniform flow can be calculated using a standard form of Stokes' theorem:

$$
\mathbf{F}_{H D}=-6 \pi a \eta \mathbf{v}
$$

where $\eta$ is the fluid viscosity, $a$ is the particle radius, and $\mathbf{v}$ the particle velocity vector (with respect to the fluid).

The hydrodynamic flow within a micro-channel is predominantly laminar, the small channel dimensions and the flow rates 
used in LOC devices (typically less than $1 \mathrm{~mm} / \mathrm{sec}$ ) ensuring that the Reynolds number for the system is much less than 1 . Hence, the flow velocity of the fluid in the channel has a parabolic profile; the fluid has highest velocity in the centre of the channel, and is close to zero near to the walls. A particle within such a fluid is within a shearing flow, the magnitude of the shear depends on the position of the particle with respect to the wall. Stokes' theorem can be modified to determine the hydrodynamic drag on a spherical particle in a shear flow:

$$
\mathbf{F}_{S, H D}=-6 \pi a \eta h S
$$

where $h$ is the height of the particle within the shear field, and $S$ is the shear rate within the flow. Such a calculation assumes that the flow around the particle is unrestricted, however, and becomes unreliable for a particle near to a plane wall. Goldman et al. ${ }^{23}$ found that wall effects increased the hydrodynamic drag on a spherical particle in a laminar shear flow, and the effect could be modelled by a non-dimensional coefficient that is proportional to the distance of the particle from the wall:

$$
\mathbf{F}_{W, H D}=-6 \pi a \eta h S K
$$

where $K$ is a coefficient that incorporates wall effects, and for the case where the particle is in contact with the wall $(h / a=1)$, this coefficient has a value of 1.7005 .

The velocity profile is determined from the solution of the Navier-Stokes equation:

$$
\rho_{m} \frac{d \mathbf{u}}{d t}+\rho_{m}(\mathbf{u} \cdot \nabla) \mathbf{u}=-\nabla p+\eta \nabla^{2} \mathbf{u}+\boldsymbol{f}
$$

where $\rho_{m}$ is the fluid density, $\mathbf{u}$ is the fluid velocity, $\nabla p$ the pressure gradient along the channel, and $\boldsymbol{f}$ is the body force on the fluid. For a steady, unidirectional flow (defined as along the $x$ axis), this equation reduces to the form:

$$
\nabla^{2} \mathbf{u}=\frac{1}{\eta} \frac{\partial p}{\partial x}
$$

The flow profile in a duct or channel of rectangular crosssection can therefore be found by solving Poisson's equation, as demonstrated using a Fourier series expansion in the ESI. $\dagger$

\section{Materials and methods}

\section{Device fabrication}

The electrodes were fabricated on $150 \mathrm{~mm}$ diameter, $700 \mu \mathrm{m}$ thick glass wafers. Electrode layers were made from layers of titanium (for adhesion) and platinum, patterned using photolithography and ion beam milling. As mentioned previously, in order to fabricate a ring electrode in the ground plane, two metal layers separated by a dielectric insulator are required. The dielectric was a $1 \mu \mathrm{m}$ thick layer of benzocyclobutene (BCB) patterned using reactive ion etching. Wafers were diced into individual chips, $20 \mathrm{~mm}$ square. The ring electrodes were fabricated with internal diameters of 40 and $80 \mu \mathrm{m}$, the width of the ring electrode was $10 \mu \mathrm{m}$ and the gap between the ring and the ground plane was $10 \mu \mathrm{m}$.

The microfluidic channel was fabricated separately on each chip, from a layer of Ordyl SY355 dry film resist (Elga Europe), bonded between the chip and a glass lid. One layer of resist was laminated on to each of the two surfaces (chip and glass lid) by hot-rolling at $100{ }^{\circ} \mathrm{C}$. The laminate was patterned by exposure to UV radiation through a negative contact mask and developed in BMR developer (Elga Europe) using a process similar to that described by Vulto et al. ${ }^{24}$ The height of the bonded channel was $100 \mu \mathrm{m}$. A closed microfluidic channel was produced by bonding the two resist layers together at $200{ }^{\circ} \mathrm{C}$. Inlet and outlet holes $(1$ $\mathrm{mm}$ diameter) were drilled in the glass lid after bonding.

\section{Experimental}

A microfluidic manifold was used to interface macroscale fluidic connections to the microdevice and also provided electrical contact via spring contacts mounted on a printed circuit board. Bead suspension was driven through the device using a ColePalmer 79000 syringe pump with flow rates in the range 0.25 to $20 \mu 1 / \mathrm{min}$.

Latex test particles were suspended in a solution of $0.1 \mathrm{mM}$ $\mathrm{KCl}$ containing $0.02 \%$ (v/v) TWEEN-20, prepared in deionised water. The conductivity was measured to be $1.9 \mathrm{mS} / \mathrm{m}\left(25^{\circ} \mathrm{C}\right)$ using a (Hanna EC215) conductivity meter. Polystyrene microspheres (Polybeads, Polysciences Ltd) were purchased from Park Scientific Inc, and had a mean diameter of $15.61 \mu \mathrm{m}(\mathrm{CV} \leq 15 \%$, density 1.05.). For trap characterisation, a $100 \mu$ l aliquot of bead suspension $\left(1.35 \times 10^{7}\right.$ beads $/ \mathrm{ml}$, or $2.5 \%$ solids $)$ was washed three times in the $0.1 \mathrm{mM} \mathrm{KCl} / \mathrm{TWEEN}$ solution by centrifugation and resuspension. Bead solutions were passed through a $41 \mu \mathrm{m}$ filter (Whatman) prior to use. Particles were imaged and tracked using a home-made fluorescence microscope, built around a Nikon $10 \times$ Plan Fluor objective lens and a Panasonic AW-E600E colour camera. A blue LED (Lumiled Luxeon, peak output $470 \mathrm{~nm}$ ) provided illumination for (FITC/GFP compatible) fluorescence observations, while broadband illumination from a 'white' LED (5500K CCT) mounted underneath the target was used for transmitted-light measurements.

\section{Modelling and simulation}

Electric fields were solved numerically using finite element analysis software (Comsol Multiphysics 3.4, Comsol Ltd). In the case of the ring electrode, the field distribution in a plane normal to the substrate and through the centre of the trap was modelled in cylindrical geometry using the electrostatic form of Poisson's equation. The upper boundary was set to be a perfect insulator (zero normal current). Although in reality this is a glass substrate but the very large difference in conductivity between fluid and glass, makes this assumption valid up to the charge relaxation frequency of the system (300 MHz in PBS). ${ }^{25}$ This issue is discussed in the ESI $\dagger$ in more detail, demonstrating that for the range of experimental frequencies and conductivities used in this work, the low frequency electrostatic simulation of the trap is accurate.

\section{Results and discussion}

\section{Numerical calculation of the field}

The electric field from the electrode array was simulated for an applied potential of $1 \mathrm{~V}$ between the ring and the surrounding 


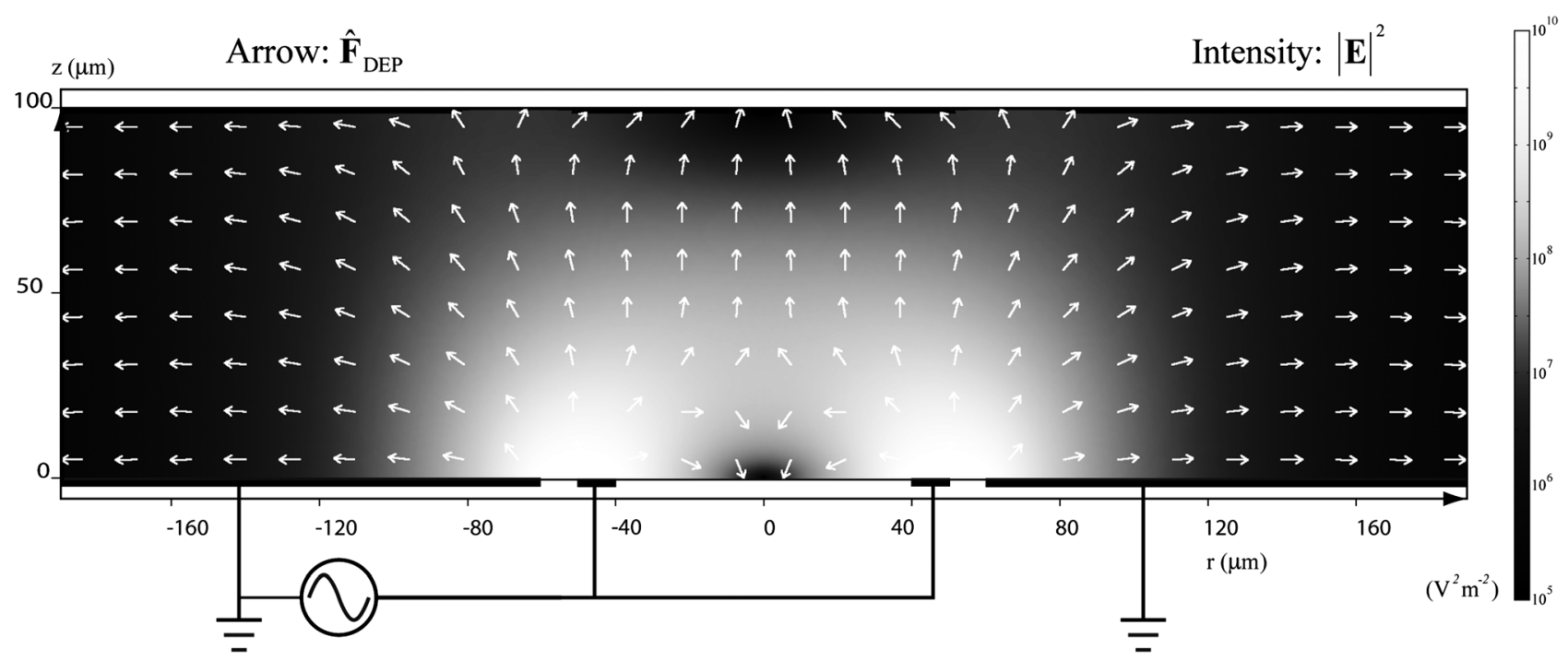

Fig. 2 Schematic cross-section of the ring trap showing the electrodes and the applied potential, with a gray-scale plot showing the magnitude of the electric field squared; proportional to the DEP potential energy. The arrows are normalised vectors and indicate the direction of the nDEP force (not the magnitude). Particles are trapped by nDEP in regions of low field strength: there is a strong trap in the centre of the ring at the lower substrate and a second weaker trap at the upper glass surface. As discussed in the ESI, $\dagger$ the upper trap is significantly weaker.

plane. Only the top metal layer was simulated, neglecting the other layers buried beneath the dielectric. The roof of the flow chamber and the substrate surface between the electrodes were set as insulating boundaries, and the chamber height was $100 \mu \mathrm{m}$. The simulation was performed at the low-frequency electrostatic limit (see ESI†). Fig. 2 shows a plot of the square of the electric field magnitude $\left(\mathrm{E}^{2}\right)$, which is proportional to the potential energy for the dielectrophoretic force, as a grayscale plot with the direction of the DEP force (nDEP) indicated by the vectors. Traps and cages are regions of low field magnitude entirely surrounded by higher values of field strength, to which the nDEP vectors point. As can be seen in this figure, there are two such regions in this electrode design, one in the centre of the ring electrode and a second, very weak trap on the roof of the chamber directly above the ring. As this is a cross-section of a system with rotational symmetry, this plot represents the entire solution of the field, and the trap is in fact a closed cage in 3D. When the cage is switched on, with a particle inside the ring, the particle will be pushed down and into the centre of the ring. Simultaneously, all other particles are repelled from the ring, as shown by the vector plot in the figure. The simulation also shows that the force keeping a particle centred in the trap increases as the particle moves closer to the edge.

\section{Trapping single particles}

Fig. 3 shows still images from a video of $15 \mu \mathrm{m}$ diameter polystyrene particles trapped against a fluid flow for (a) an $80 \mu \mathrm{m}$ diameter ring and (b) four $40 \mu \mathrm{m}$ rings from a larger array. In each case the excitation voltage was $5 \mathrm{~V}$ peak to peak @ $1 \mathrm{MHz}$. Particles could be held against fluid flow rates of up to $5.5 \mu \mathrm{l} / \mathrm{min}$ in the $80 \mu \mathrm{m}$ cage and up to $20 \mu \mathrm{l} / \mathrm{min}$ in the $40 \mu \mathrm{m}$ cage. Particles were never observed to be trapped at the roof of the device even at very low flows. Particles held in the $80 \mu \mathrm{m}$ cage were displaced from the centre of the trap as the fluid flow rate increased. This displacement was measured to calculate the dielectrophoretic force in the cage - see later.

\section{Characterisation of the trapping force}

As shown in Fig. 2, the electric field strength varies greatly across the centre of the ring array, with a zero value in the centre and maximum at the electrode edges in the gap between the electrodes. For a given height, the lateral DEP force is zero in the centre, and increases to a maximum over the ring electrode. When a trapped particle is subjected to a fluid flow, then under steady-state conditions the particle is displaced a certain distance from the centre of the ring. This position is given by the balance of the Stokes drag force and the DEP trapping force. Therefore the DEP force was determined by varying the fluid flow velocity and measuring the displacement of the particle within the ring.

A bead suspension was pumped through the channel, and a single bead immobilised in a ring trap using a signal of $1 \mathrm{MHz}$ at $5 \mathrm{~V}$ peak-peak. With a bead trapped, the flow rate was increased in steps from 0 to $5.5 \mu \mathrm{l} / \mathrm{min}$, and the position of the bead recorded. Data was recorded for 10 seconds for each flow rate, and 20 frames from each clip at 0.5 second intervals were analysed. Bead position relative to the centre of the trap was measured (in pixels, and converted to $\mu \mathrm{m}$ ) for each frame, and an average value for all 20 frames was obtained. This experiment was repeated four times. The data is plotted in Fig. 4A, showing bead displacement against volumetric flow rate. The displacement from the centre of the array increases with increasing flow rate, but the rate of increase slows as the particle approaches the ring due to the rapidly increasing DEP force. At an applied voltage of $5 \mathrm{~V}$ peak-peak, the beads escaped from the trap when the flow rate exceeded $5.5 \mu \mathrm{l} / \mathrm{min}$.

For comparison with experimental data, the force on a 15.6 $\mu \mathrm{m}$ diameter polystyrene particle was calculated using equation (1) and the simulated electric field, setting $\operatorname{Re}\left(f_{C M}\right)=-0.475$ 

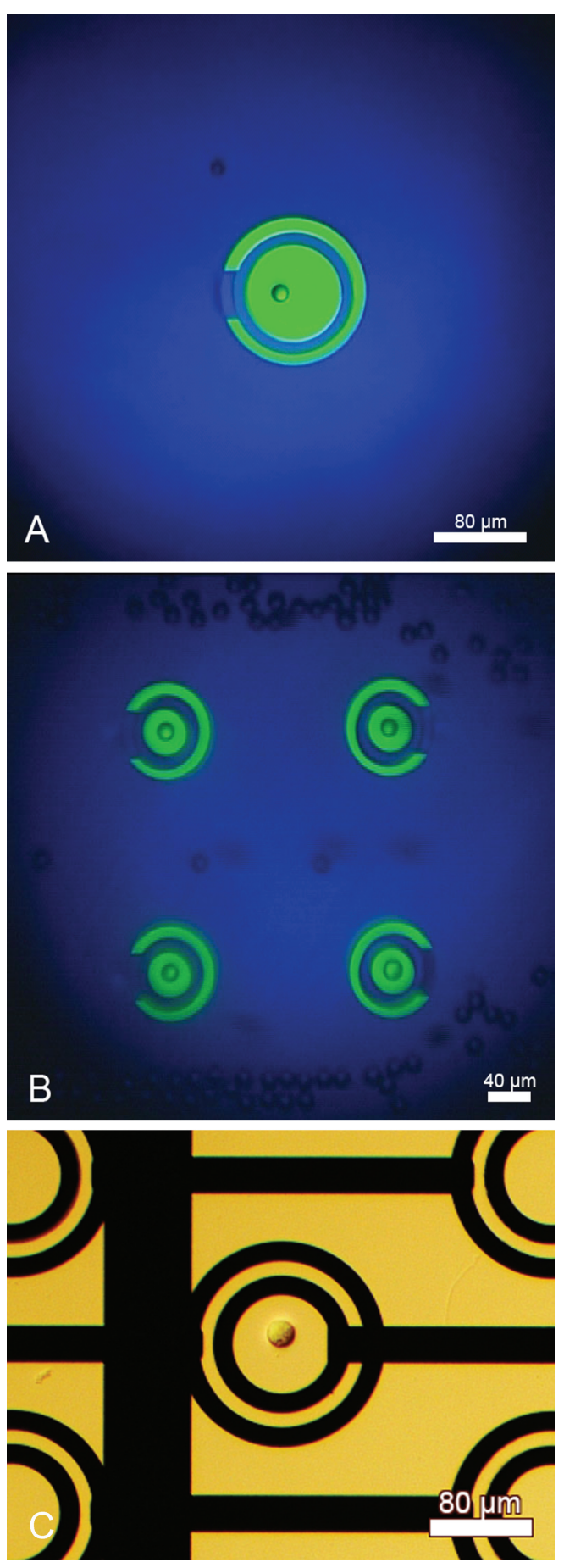

Fig. 3 (A \& B) Still images taken from video showing the individual trapping of single $15 \mu \mathrm{m}$ diameter polystyrene beads in (A) a $80 \mu \mathrm{m}$ diameter ring trap and (B) four $40 \mu \mathrm{m}$ diameter ring traps from a larger array of up to 48 traps. The repulsion of the remaining beads, keeping them from entering the traps can also be seen in (B). A video is included in the ESI. $\uparrow$ (C) A single HeLa cell immobilised in a nDEP ring trap against a continuous flow (of DMEM culture medium) at $1.0 \mu \mathrm{l} / \mathrm{min}$. Electrical excitation is $2.5 \mathrm{~V}$ peak at $20 \mathrm{MHz}$.

(with $\varepsilon_{\mathrm{r}}=2.5, \sigma_{p}=0.27 \mathrm{mS} / \mathrm{m}, V=5 \mathrm{~V}$ peak-peak and $f=1$ $\mathrm{MHz}$ ). Only the horizontal component of the DEP force is considered, as this is the only component that can be determined
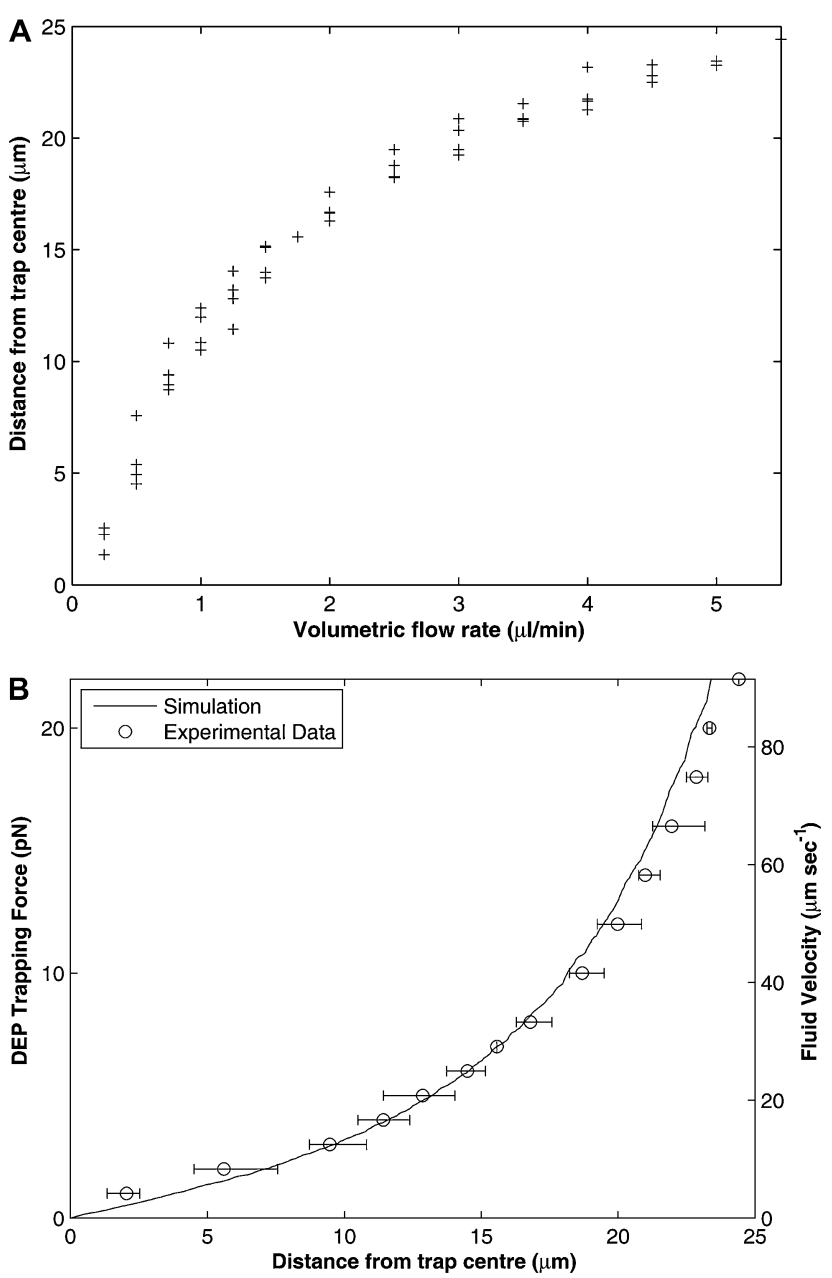

Fig. 4 (A) The measured displacement from the trap centre at different applied flow rates of $15 \mu \mathrm{m}$ diameter polystyrene beads trapped in the centre of an $80 \mu \mathrm{m}$ ring trap for an applied voltage of $5 \mathrm{~V}$ peak to peak at $1 \mathrm{MHz}$. (B) A plot of the DEP trapping force determined from the fluid velocity (shown on the right-hand axis) against displacement from the trap centre for the data shown in (A). Also shown is the dielectrophoretic force calculated from the simulated field demonstrating good agreement.

directly from the hydrodynamic drag force. The results are plotted as a line in Fig. 4B. Also shown are the values of the trapping force determined from the experimental data, assuming that it is balanced by the modified Stokes drag force, calculated using equation 6 and the volumetric flow rate. The velocity of the fluid is shown on the opposite axis; as the particle is in a shear flow this is the velocity impinging on the centre of the particle. Comparison of the experimental data with the simulated force shows excellent agreement $\left(\mathrm{R}^{2}=0.9807\right)$, with small deviations in the centre and edge of the trap. The discrepancy at small displacements may be due to errors in measurement of small displacements and the difficulty in controlling low flow rates. At the edge of the trap the error may be due to the limitations of the dipole approximation used to calculate the force. Equally, a near perfect agreement $\left(\mathrm{R}^{2}=0.9966\right)$ is obtained if the applied voltage used in the simulation is reduced to $4.8 \mathrm{~V}$ peak-peak, suggesting that a small voltage drop could have occurred along the interconnects. 


\section{Trapping single cells}

The ring traps were also used to trap cells suspended in a physiological medium. HeLa (Human epithelial carcinoma) cells were cultured in DMEM (Dulbecco's Modified Eagle's Medium - 4mM L-glutamine, Hepes buffer, no Pyruvate) with $10 \%$ foetal calf serum and $100 \mu \mathrm{g} / \mathrm{ml}$ Penicillin/Streptomycin, at $37{ }^{\circ} \mathrm{C}$. To maintain growth, the cultures were split every $3 \mathrm{rd}$ or 4 th day by trypsinisation, and fresh culture medium added. For experiments, the cells were removed from culture, incubated at $37{ }^{\circ} \mathrm{C}$ and used within 3 hours. The cells were concentrated by centrifugation in culture medium to a density of $10^{6}$ cells $/ \mathrm{ml}$. For the cell trapping a slightly different and simpler trap design was used, where the ground plane was replaced by a second ring (diameter: $120 \mu \mathrm{m}$ internal, $140 \mu \mathrm{m}$ external) - Fig. 3C. These electrodes produced the same trapping forces (up to $27.5 \mathrm{pN}$ on a $15.6 \mu \mathrm{m}$ diameter latex particle, $5 \mathrm{Vpp} @ 1 \mathrm{MHz}$ compared with $23 \mathrm{pN}$ for the previous design). The microfluidic channel was $700 \mu \mathrm{m} \times 100 \mu \mathrm{m}$, so lower volumetric flow rates were required to produce equivalent Stokes drag. Prior to use, the chip was flushed through with DMEM, and a sample of HeLa cell suspension injected at a flow rate of $10 \mu \mathrm{l} / \mathrm{min}$. Fig. 3(C) shows a captured image of a trapped single Hela cell. The ring electrode cage could hold the cell against a fluid flow of $1.03 \mu \mathrm{l} /$ min (mean value, s.d. $=0.11$ ) with an applied signal of $5 \mathrm{~V}$ peak-peak at $20 \mathrm{MHz}$. This corresponds to a trapping force of $13.8 \mathrm{pN}$ for a $15.9 \mu \mathrm{m}$ cell (mean value, s.d. $=1.2$ ). The smaller force on the cells is due to the lower value of the ClausiusMossotti factor for the cells compared with the beads; also the double layer on the electrode reduces the potential in the bulk at this frequency in DMEM.

\section{Discussion}

A major challenge in designing large-area array traps is optimising the electrical connectivity to each trap. The single layer trap described by Rosenthal et al. ${ }^{14}$ was fabricated from a single layer of metal. The system consisted of a square electrode adjacent to a ground line and is simple to fabricate as only a single metal layer needs to be patterned. But this design does not allow control over individual traps. An active matrix architecture can resolve these issues; each individual electrode is driven by an integrated solid-state switch, permitting active matrix addressing techniques to be used, i.e. an $(m \times n)$ matrix can be addressed with $(m+n)$ connections. ${ }^{15,17}$ Power dissipation can become a critical issue as the number of traps increases, or the area of electrodes is scaled up, particularly when high conductivity media is required. The design of microelectrode structures must be optimised to minimise power dissipation if they are intended to be used for the manipulation of cells in high conductivity media (such as culture medium or PBS). Confining the field to regions where particle manipulation is required is useful for controlling the power dissipation into the fluid. A dielectric layer covering regions of the electrodes not involved in particle manipulation (such as connecting tracks) confines the electric field and reduces extraneous power dissipation in the fluid, and also stops dielectrophoretic effects outside the region of intended manipulation. The radially symmetric trap architecture described in this paper permits trapping and release of particles from any direction, compared with the square traps. ${ }^{14,18}$ They are also suitable for integration into an active matrix CMOS or TFT driven system to create large area dynamically addressable cell trap platforms.

If electrokinetics is to be used in devices for cell manipulation and culture, then cell viability must be maintained. An electrical potential develops across the highly insulating cellular membrane, and this depends on the applied frequency, as well as voltage and suspending medium conductivity. The HeLa cells were immobilised using a signal of $20 \mathrm{MHz}$ at $5 \mathrm{~V}$ peak-peak. Calculations $^{26-28}$ indicate the potential drop across the membrane would be approximately $0.3 \mathrm{mV}$ - well below the threshold at which damage to the membrane is likely to occur. Simulation of Joule heating predicts a temperature rise of 3.6 ${ }^{\circ} \mathrm{C}$ in the centre of an isolated trap (@ $5 \mathrm{Vpp}, 20 \mathrm{MHz}, \sigma_{\text {media }}=$ 1.6 S/m), increasing to $12{ }^{\circ} \mathrm{C}$ for a densely populated array of traps $(190 \mu \mathrm{m}$ pitch). This model assumes the glass substrates are cooled in air at $295 \mathrm{~K}$. The temperature rise is significantly reduced if the substrate is cooled, for example at 10 volts peak to peak the temperature can be maintained at $20{ }^{\circ} \mathrm{C}$ in the vicinity of the cell, if the substrate base is cooled to $4{ }^{\circ} \mathrm{C}$ (see $\mathrm{ESI} \dagger$ ).

The maximum trapping force developed on a $15.6 \mu \mathrm{m}$ latex bead was $23 \mathrm{pN}$, sufficient to immobilise the particle against a flow of $5.5 \mu \mathrm{l} / \mathrm{min}$. To put this into context, this exceeds the particle's weight force of $20.48 \mathrm{pN}$ (assuming density $=1.05 \mathrm{~g}$ / $\mathrm{ml}$, particle mass $=2.09 \times 10^{-12} \mathrm{~kg}$ ). Hence, in the absence of a flow, the particle would remain trapped if the trap array were to be held vertically. The DEP trapping force scales with the third power of particle radius $\left(\mathrm{a}^{3}\right.$, equation 1$)$, while the hydrodynamic drag scales with the first power of radius (equation 4). This means that larger particles can be trapped at higher flow rates than smaller particles, for a given value of applied voltage and trap size. The maximum flow rate against which biological cells can be held is generally lower than for similarly sized polystyrene particles, because the ClausiusMossotti factor for cells suspended in physiological media is lower than for polystyrene particles at frequencies suitable for nDEP.

\section{Conclusions}

We have designed and characterised a new nDEP trap that can be used to selectively hold single cells against a fluid flow. We have shown that a $15.6 \mu \mathrm{m}$ latex bead can be held in the trap against a flow of up to $5.5 \mu \mathrm{l} / \mathrm{min}$, producing a force of $23 \mathrm{pN}$. HeLa cells could also be trapped, and a force of $13.8 \mathrm{pN}$ was produced. The traps are individually controllable and suitable for arrayed operation, and are compatible with physiological media suitable for cell culture. With the addition of a suitable detection mechanism (such as fluorescence or impedance spectroscopy) together with control systems, applications include rare cell isolation, cell patterning, or temporally distributed cytometric measurements.

\section{Acknowledgements}

R. Thomas would like to thank Philips Research Laboratories (UK) for a CASE award. We also acknowledge Philips MiPlaza 
in Eindhoven, NL for fabrication of the electrode structures on glass. We thank Charlotte Hague, University of Southampton for the HeLa cells.

\section{References}

1 P. J. Hung, P. J. Lee, P. Sabounchi, R. Lin and L. P. Lee, Biotechnology and Bioengineering, 2005, 89(1), 1-8.

2 K. Dholakia, P. Reece and M. Gu, Chemical Society Reviews, 2008, 37(1), 42-55.

3 D. Di Carlo, L. Y. Wu and L. P. Lee, Lab on a Chip, 2006, 6(11), 1445-1449.

4 F. F. Becker, X. B. Wang, Y. Huang, R. Pethig, J. Vykoukal and P. R. C. Gascoyne, Journal of Physics D-Applied Physics, 1994, 27(12), 2659-2662.

5 D. Holmes, N. G. Green and H. Morgan, IEEE Engineering in Medicine and Biology Magazine, 2003, 22(6), 85-90.

6 M. S. Talary, J. P. H. Burt, J. A. Tame and R. Pethig, Journal of Physics D-Applied Physics, 1996, 29(8), 2198-2203.

7 U. Seger, S. Gawad, R. Johann, A. Bertsch and P. Renaud, Lab on a Chip, 2004, 4(2), 148-151.

8 S. Fiedler, S. G. Shirley, T. Schnelle and G. Fuhr, Analytical Chemistry, 1998, 70(9), 1909-1915.

9 F. F. Becker, X. B. Wang, Y. Huang, R. Pethig, J. Vykoukal and P. R. C. Gascoyne, Proceedings of the National Academy of Sciences of the United States of America, 1995, 92(3), 860-864.

10 D. S. Gray, J. L. Tan, J. Voldman and C. S. Chen, Biosensors \& Bioelectronics, 2004, 19(7), 771-780.

11 A. Menachery and R. Pethig, IEE Proceedings-Nanobiotechnology, 2005, 152(4), 145-149.

12 J. El-Ali, P. K. Sorger and K. F. Jensen, Nature, 2006, 442(7101), 403411.
13 P. S. Dittrich and A. Manz, Nature Reviews Drug Discovery, 2006, 5(3), 210-218.

14 A. Rosenthal and J. Voldman, Biophysical Journal, 2005, 88(3), 21932205.

15 N. Manaresi, A. Romani, G. Medoro, L. Altomare, A. Leonardi, M. Tartagni and R. Guerrieri, IEEE Journal of Solid-State Circuits, 2003, 38(12), 2297-2305.

16 T. Schnelle, R. Hagedorn, G. Fuhr, S. Fiedler and T. Muller, Biochimica et Biophysica Acta, 1993, 1157(2), 127-140.

17 T. P. Hunt, D. Issadore and R. M. Westervelt, Lab on a Chip, 2008, 8(1), 81-87.

18 N. Mittal, A. Rosenthal and J. Voldman, Lab on a Chip, 2007, 7(9), 1146-1153.

19 H. A. Pohl. Dielectrophoresis, Cambridge University Press, 1978.

20 T. B. Jones. Electromechanics of Particles, Cambridge University Press, 1995.

21 H. Morgan, N. G. Green. AC Electrokinetics: Colloids and Nanoparticles, Research Studies Press, 2003.

22 J. Voldman, Annual Review of Biomedical Engineering, 2006, 8, 425454.

23 A. J. Goldman, R. G. Cox and H. Brenner, Chemical Engineering Science, 1967, 22(4), 653-660.

24 P. Vulto, N. Glade, L. Altomare, J. Bablet, L. Del Tin, G. Medoro, I. Chartier, N. Manaresi, M. Tartagni and R. Guerrieri, Lab on a Chip, 2005, 5(2), 158-162.

25 N. G. Green, A. Ramos, A. Gonzalez, A. Castellanos and H. Morgan, Journal of Electrostatics, 2001, 53(2), 71-87.

26 C. Grosse and H. P. Schwan, Biophysical Journal, 1992, 63(6), 16321642 .

27 S. Archer, T. T. Li, A. T. Evans, S. T. Britland and H. Morgan, Biochemical and Biophysical Research Communications, 1999, 257(3), 687-698.

28 K. Asami, Y. Takahashi and S. Takashima, Biophysical Journal, 1990, 58(1), 143-148. 